\title{
Conformation Changes and Subsequent Gelation of Conalbumin by a Thiol Reagent
}

\author{
Hideo OE, Masaaki Hirose and Etsushiro DoI \\ The Research Institute for Food Science, Kyoto University, \\ Uji, Kyoto 611, Japan \\ Received March 19, 1986
}

\begin{abstract}
Thiol reagents have been shown to induce the gelation of conalbumin [M. Hirose, H. Oe and E. Doi, Agric. Biol. Chem., 50, 59 (1986)]. The gelation mechanisms were investigated in relation to the structure of this protein. Following the addition of 2-mercaptoethanol, the sulfhydrỳl groups of conalbumin increased almost linearly during the first $50 \mathrm{~min}$ of incubation. The relative hydrophobicity measured by fluorescent dyes increased concomitantly with increases in the sulfhydryl groups. The circular dichroism spectra revealed that the thiol reagent induces some changes in the tertiary structure but no prominent alterations in the secondary structure. The transmission electron microscopic study showed that at $20 \mathrm{~min}$ of incubation conalbumin formed rod-like structures and that at $32 \mathrm{~min}$ of incubation these structures produced branched aggregates. The SDS-polyacrylamide gel electrophoresis indicated that intermolecular covalent interactions are not involved in the formation of conalbumin gel.
\end{abstract}

In our previous work, ${ }^{1)}$ we found that thiol reagents such as 2-ME and cysteine induce the gelation of egg white at low temperatures and have concluded that conalbumin is a major component in this gelation of egg white. It is known that conalbumin contains 30 halfcystine residues in one molecule, all of which exist as 15 disulfide linkages. ${ }^{2)}$ Therefore, it was suggested that the thiol-dependent gelation is closely correlated with the cleavage of some disulfide linkages in the conalbumin molecule. Preliminarily we found that during the incubation with 2-ME the gelation of conalbumin begins after a lag of about $60 \mathrm{~min}$. This paper mainly deals with the changes in conalbumin structure during this lag to elucidate the mechanisms for the thiol-dependent gelation of this protein. The data show that the addition of 2-ME increases both sulfhydryl content and hydrophobicity in conalbumin molecules. These increases were followed by the formation of an aggregated structure which was observed with transmission electron micros- copy. We also investigated, using SDS-polyacrylamide gel electrophoresis, whether intermolecular covalent interaction was involved in the conalbumin gelation.

\section{MATERIALS AND METHODS}

Materials. cis-PA and ANS were obtained from P. L. Biochemicals Inc. and from Wako Pure Chemical Industries Ltd., respectively. DTNB, sodium iodoacetate, and other chemicals were obtained from Nakarai Chemicals Ltd.

Conalbumin was purified from the post-ovalbumin supernatant as described previously. ${ }^{1)}$ Conalbumin in $10 \mathrm{~mm}$ Tris- $\mathrm{HCl}$ buffer, $\mathrm{pH} 8.0$, was stored at $-20^{\circ} \mathrm{C}$ until used. Protein purity was checked by SDS-electrophoresis in polyacrylamide gels.

Conditions for the incubation with 2-ME. Unless otherwise stated, the reaction mixture consisted of $1.6 \%$ conalbumin, $70 \mathrm{~mm} 2-\mathrm{ME}$, and $0.1 \mathrm{M}$ sodium phosphate buffer, $\mathrm{pH}$ 8.0. After incubation at $35^{\circ} \mathrm{C}$ for the indicated periods, the sample was analyzed by turbidity, sulfhydryl content, hydrophobicity, circular dichroism, transmission electron microscopy, and SDS-gel electrophoresis.

Abbreviations: ANS, 8-anilino-1-naphthalene sulfonate; DTNB, 5,5'-dithiobis(2-nitrobenzoic acid); EDTA, ethylenediamine tetraacetic acid; 2-ME, 2-mercaptoethanol; cis-PA, cis-parinaric acid; SDS, sodium dodecyl sulfate. 
Measurement of turbidity. Turbidity of the conalbumin sample was measured at $35^{\circ} \mathrm{C}$ with a Shimadzu UV visible recording spectrophotometer UV-240 at $600 \mathrm{~nm}$.

Measurement of the content of sulfhydryl group. The sulfhydryl contents of conalbumin during the incubation with 2-ME were measured by the photometric method of Ellman. ${ }^{3)}$ Conalbumin in $50 \mu \mathrm{l}$ of the reaction mixture was precipitated at different times with $5 \mathrm{ml}$ of cold acetone$\mathrm{HCl}$ solution (one drop of conc. $\mathrm{HCl} / 10 \mathrm{ml}$ of acetone). After centrifuged at $2,000 \times g$ for $10 \mathrm{~min}$, the precipitate was washed 3 times with $5 \mathrm{ml}$ of acetone. The precipitate was dried in vacuo at $50^{\circ} \mathrm{C}$ and dissolved in $2 \mathrm{ml}$ of $0.1 \mathrm{M}$ sodium phosphate, $\mathrm{pH} 8.0$, containing $0.48 \% \mathrm{SDS}, 8 \mathrm{M}$ urea, and $1 \mathrm{~mm}$ EDTA at $50^{\circ} \mathrm{C}$ for a few min. To the dissolved sample, $10 \mu \mathrm{l}$ of $10 \mathrm{~mm}$ DTNB in $0.1 \mathrm{M}$ sodium phosphate, $\mathrm{pH} 7.0$, was added. Then $2 \mathrm{ml}$ of distilled water was added to the sample and its absorbance was measured at $412 \mathrm{~nm}$. The sulfhydryl content was calculated using the molar extinction coefficient of $13,600 \mathrm{M}^{-1} \mathrm{~cm}^{-1}$.

The sulfhydryl content in conalbumin gel was also measured by amino acid analysis. The conalbumin gel obtained by the $24-\mathrm{hr}$ incubation at $35^{\circ} \mathrm{C}$ was extensively crushed with a glass rod and centrifuged at $2,000 \times g$ for $10 \mathrm{~min}$. The crushed gel matrices (about $1 \mathrm{mg}$ as protein) was washed 3 times with $0.1 \mathrm{~m}$ sodium phosphate, $\mathrm{pH} 8.0$, containing $1 \mathrm{~mm}$ EDTA, dissolved in $1.0 \mathrm{ml}$ of $0.1 \mathrm{M}$ sodium phosphate, $\mathrm{pH} 8.0$, containing $8 \mathrm{M}$ urea and $1 \mathrm{mM}$ EDTA. After incubation with $20 \mathrm{~mm}$ sodium iodoacetate at $35^{\circ} \mathrm{C}$ for one hour, the solution was dialyzed against distilled water at $4^{\circ} \mathrm{C}$ for 3 days. The sample was lyophilized and hydrolyzed in $6 \mathrm{~N} \mathrm{HCl}$ at $110^{\circ} \mathrm{C}$ for $48 \mathrm{hr}$. $S$ Carboxymethylcysteine was quantified with a Hitachi 835 amino acid analyzer. As a comparable experiment, conalbumin was denatured by reduction and alkylation in the presence of $8 \mathrm{M}$ urea. Conalbumin $(10 \mathrm{mg})$ in $2 \mathrm{ml}$ of $50 \mathrm{~mm}$ sodium phosphate, $\mathrm{pH} 8.0$, containing $8 \mathrm{M}$ urea and $0.2 \mathrm{M}$ 2-ME was incubated at $35^{\circ} \mathrm{C}$ for one hour, and then the protein was alkylated by incubating with $300 \mathrm{~mm}$ sodium iodoacetate at $35^{\circ} \mathrm{C}$ for one hour. The reacted sample was dialyzed against distilled water and lyophilized. The $S$ carboxymethylcysteine content in the sample was measured by amino acid analysis.

Measurement of hydrophobicity. The relative hydrophobicity was measured fluorimetrically using cis-PA ${ }^{4)}$ and ANS. ${ }^{5)}$ cis-PA was dissolved in ethanol purged with nitrogen to make a concentration of $3.6 \mathrm{~mm}$. The cis-PA solution contained $3.6 \mathrm{mM}$ 2,6-di-tert-butyl-p-cresol as an antioxidant. ANS (1 mM) was dissolved in deoxidized water. Samples, each $5 \mu \mathrm{l}$, of the reaction mixture at various times were diluted with $3 \mathrm{ml}$ of $10 \mathrm{~mm}$ sodium phosphate, pH 7.4. Fifteen $\mu \mathrm{l}$ of $c i s$-PA solution or $20 \mu \mathrm{l}$ of ANS solution was added to the diluted reaction mixture. Fluorescence intensity was measured at $420 \mathrm{~nm}$ for $c i s$-PA and at $470 \mathrm{~nm}$ for ANS with a Hitachi MPF-4 fluorescence spectrophotometer. Excitation wavelengths were $325 \mathrm{~nm}$ and $400 \mathrm{~nm}$ for cis-PA and ANS, respectively.

Circular dichroism spectra. The circular dichroism (CD) spectra were recorded using a Jasco spectropolarimeter J$5000 \mathrm{C}$ with a data processor Model DP-501. The native or 2-ME-reacted conalbumin was diluted 20 times with $0.1 \mathrm{~m}$ sodium phosphate, $\mathrm{pH}$ 8.0. The $\mathrm{CD}$ data in the $190 \sim 245 \mathrm{~nm}$ regions were expressed in terms of the mean molecular ellipticity $[\theta]^{\mathrm{MRW}}$, taking 111 as the mean residue weight calculated from the data of Williams et al. ${ }^{2)}$ and in the $245 \sim 340 \mathrm{~nm}$ regions as ellipticity per mole of conalbumin, $[\theta]$. All of the $\mathrm{CD}$ spectra were corrected by subtracting the spectrum in the absence of the protein. The contents of $\alpha$-helix and $\beta$-structure were calculated by the CD program of Hennessey and Johnson. ${ }^{6}$

Transmission electron microscopy. The reaction mixture was diluted $80 \sim 800$-fold with distilled water passed through a $0.45-\mu \mathrm{m}$ millipore filter. One drop was applied to a carbon-coated electron microscope grid, then a drop of negative stain, $2 \%$ potassium phosphotungstate, $\mathrm{pH}$ 7.0 was added. After it was dried on filter paper, the grid was examined in a Hitachi electron microscope $\mathrm{H}-700 \mathrm{H}$ at an accelerating voltage of $100 \mathrm{kV}$.

SDS-polyacrylamide gel electrophoresis. The conalbumin gel obtained by the 24-hr incubation with 2-ME was crushed and washed in the same way as in measurement of the content of sulfhydryl groups. The resulting gel was dissolved in $50 \mathrm{~mm}$ sodium phosphate, $\mathrm{pH} 8.0$, containing $8 \mathrm{M}$ urea and $1 \mathrm{~mm}$ EDTA, at $35^{\circ} \mathrm{C}$ for $10 \mathrm{~min}$. To a part of the dissolved solution (about $1 \mathrm{mg}$ as protein per $\mathrm{ml}$ ), sodium iodoacetate was added at a final concentration of $2 \mathrm{~mm}$, then the solution was incubated for one hour at $35^{\circ} \mathrm{C}$. Fifty $\mu \mathrm{l}$ of the conalbumin sample incubated with 2 $\mathrm{ME}$ for $35 \mathrm{~min}$ or $80 \mathrm{~min}$ was added to $0.7 \mathrm{ml}$ of the sodium phosphate buffer as described above, and then the diluted sample was incubated with $5 \mathrm{~mm}$ sodium iodoacetate for $1 \mathrm{hr}$ at $35^{\circ} \mathrm{C}$. Each sample was mixed with an equal volume of $0.125 \mathrm{M}$ Tris- $\mathrm{HCl}$ buffer, $\mathrm{pH} 7.0$, containing $2 \%$ SDS and $20 \%$ glycerol, then they were electrophoresed on $10 \%$ polyacrylamide gel as described by Laemmli. ${ }^{7)}$

Measurement of protein concentration. Protein concentration was measured by the method of Lowry et al ${ }^{8)}$ and by UV absorbance at $280 \mathrm{~nm}$ using an $E_{280 \mathrm{~nm}}^{1 \%}$ value of 12. ${ }^{9)}$ The molecular weight of conalbumin was taken as $77,770 .^{2)}$

\section{RESULTS}

Changes in turbidity, sulfhydryl content, and hydrophobicity during incubation with 2-ME As described previously, ${ }^{1)}$ the purified conalbumin formed an opaque gel with the 2-ME 


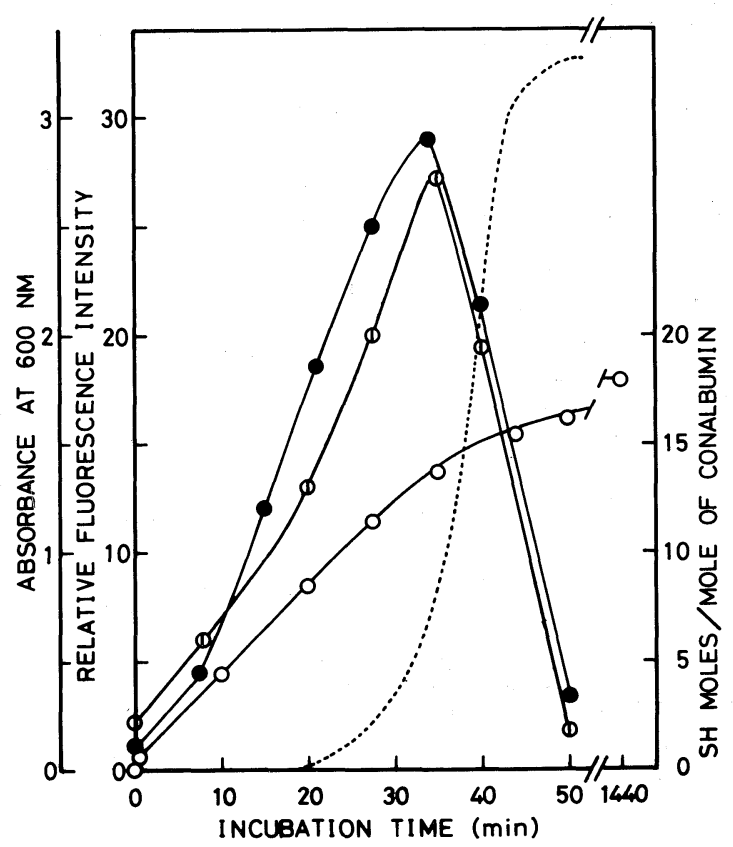

FIG. 1. Course Changes of Turbidity, Sulfhydryl Content, and Hydrophobicities of 2-ME-Reacted Conalbumin.

Conalbumin $(1.6 \%)$ in $70 \mathrm{~mm} 2-\mathrm{ME}$ and $0.1 \mathrm{M}$ sodium phosphate, $\mathrm{pH} 8.0$, was incubated at $35^{\circ} \mathrm{C}$ for various times. The turbidity of the reaction mixture was measured at $600 \mathrm{~nm}$ (-----). The sulfhydryl content and hydrophobicities of conalbumin were measured photometrically by DTNB (- - ) and fluorometrically, by cis-PA (- or ANS (-1)-), respectively.

at $35^{\circ} \mathrm{C}$ for $24 \mathrm{hr}$. The course of conalbumin gelation had a lag of about $60 \mathrm{~min}$. We observed that an increase in turbidity precedes the conalbumin gelation. As shown in Fig. 1, after a retardation time of about $20 \mathrm{~min}$, the turbidity of the conalbumin mixture gradually increased with the reaction time. Over one hour of incubation, the solution began to form a gel. We examined what kinds of changes are involved in the structure of conalbumin during the first 50-min incubation.

The native conalbumin contains 15 disulfide linkages but no free sulfhydryl residues in one molecule. ${ }^{2)}$ As shown in Fig. 1, following the addition of 2-ME, the sulfhydryl content almost linearly increased during the first $50 \mathrm{~min}$ of incubation. At $50 \mathrm{~min}$ of incubation, the number of half cystines was $16.1 \mathrm{~mol}$ per mol of conalbumin. After $24 \mathrm{hr}$ of incubation which induced a hard gel, $17.9 \mathrm{~mol}$ per mol of conalbumin were detected. When measured by amino acid analysis, the $S$-carboxymethylcysteine content of conalbumin gel was 19.2 mol per mol of protein. The $S$-carboxymethylcysteine content of the conalbumin denatured in the presence of urea and 2-ME was $30.8 \mathrm{~mol}$ per mol of conalbumin. This value was almost exactly the same as the half-cystine content found from the primary structure. ${ }^{2)}$ Therefore, our results indicate that at least nine disulfide linkages in the conalbumin molecule are cleaved by $2-\mathrm{ME}$ during the gelation process.

The relative hydrophobicity during the 50min incubation was measured by fluorophotometric methods using cis- $\mathrm{PA}^{4)}$ and $\mathrm{ANS}^{5)}$ as probes. The former compound may be effective for measuring the hydrophobicity of aliphatic amino acid groups in the protein molecule, and the latter for measuring the aromatic amino acid groups. ${ }^{10)}$ As shown in Fig. 1, the relative fluorescence intensities measured by both probes increased in parallel with increasing the sulfhydryl content and each had a maximum value at $35 \mathrm{~min}$. At this incubation time the hydrophobicity measured by cis-PA or ANS at each maximum was 26 and 12 times higher than that of native conalbumin, respectively. Further incubation resulted in large decreases in the fluorescence intensity with the increase in turbidity.

\section{Circular dichroism spectrum}

To see if the structure of conalbumin changes during the incubation with 2-ME, we did a CD study within the limited incubation time in which the data are unaffected by turbidity. The spectrum in the $190 \sim 245 \mathrm{~nm}$ regions depends on the secondary structure. As shown in Fig. 2A, the CD spectrum of native conalbumin in this region had a negative extremum at $208 \mathrm{~nm}$ and shoulders around at 215 and $233 \mathrm{~nm}$. This shape agreed well with those reported by other workers. ${ }^{11 \sim 13)}$ The shape of the CD spectrum of the conalbumin mixture obtained by the 15-min incubation with 2-ME was almost the same as that of native conalbumin. The percentages of $\alpha$ - 


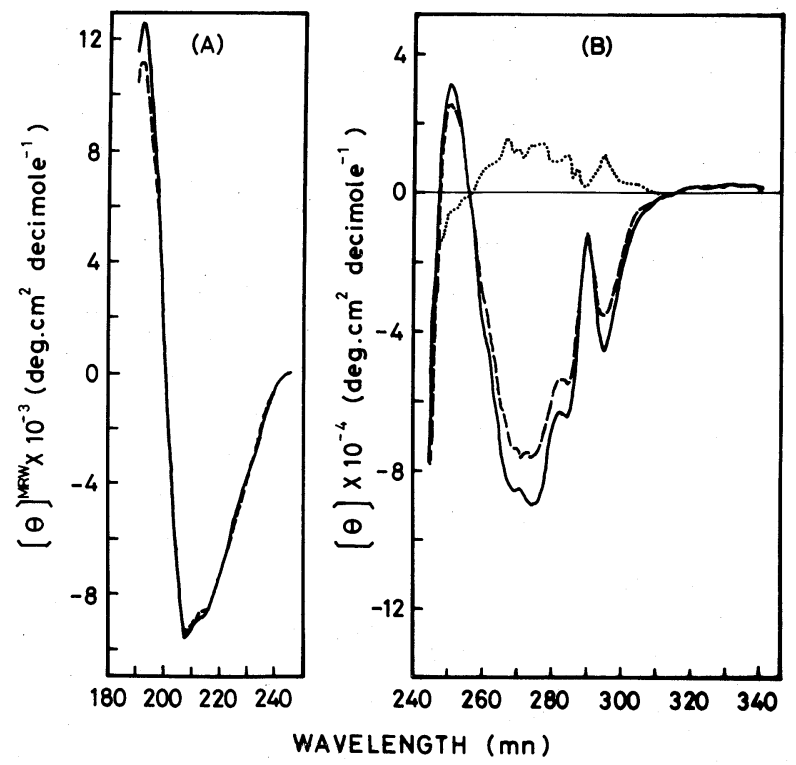

FIG. 2. CD Spectrum of Conalbumin.

The native (- ${ }_{-}$and the reacted conalbumin (---) which had been incubated with $70 \mathrm{~mm} 2-\mathrm{ME}$ for $15 \mathrm{~min}$ were diluted 20 times with $0.1 \mathrm{M}$ sodium phosphate, $\mathrm{pH} 8.0$. CD was measured in the $190 \sim 245 \mathrm{~nm}$ (A) and in the $245 \sim 340 \mathrm{~nm}$ regions (B). Each protein concentration was $1.1 \times 10^{-5} \mathrm{M}$. The optical path length of the cell was $0.02 \mathrm{~cm}$ in (A) and $1 \mathrm{~cm}$ in (B). The difference $\mathrm{CD}$ between the native and the reacted conalbumin is shown as $(\cdots)$.

Table I. Percentages of $\alpha$-Helix, $\beta$-Structure, and Random CoIl of THE NATIVE AND THE 2-ME-REACTED CONALBUMIN

The native conalbumin and the sample incubated with $70 \mathrm{~mm} 2-\mathrm{ME}$ at $35^{\circ} \mathrm{C}$ for $15 \mathrm{~min}$ were diluted. Based on the method of Hennessey and Johnson, ${ }^{6)}$ these contents of $\alpha$-helix, $\beta$-structure, and random coil were calculated.

\begin{tabular}{lccc}
\hline & $\begin{array}{c}\alpha \text {-Helix } \\
(\%)\end{array}$ & $\begin{array}{c}\beta \text {-Structure } \\
(\%)\end{array}$ & $\begin{array}{c}\text { Random coil } \\
(\%)\end{array}$ \\
\hline Native & 21.2 & 31.4 & 47.4 \\
2-ME-reacted & 25.6 & 26.3 & 48.1 \\
\hline
\end{tabular}

helix, $\beta$-structure, and random coil were calculated by the method of Hennessey and Johnson. ${ }^{6)}$ As shown in Table I, the contents of $\alpha$-helix, $\beta$-structure and randam coil of native conalbumin in $0.1 \mathrm{M}$ sodium phosphate, $\mathrm{pH} 8.0$, were $21.2,31.4$, and $47.4 \%$, respectively. The conalbumin mixture obtained by the 15-min incubation showed a slightly higher $\alpha$-helix content and a slight loss of $\beta$-structure.

The CD spectra in the $245 \sim 340 \mathrm{~nm}$ regions are contributed by the side-chain chromophores of aromatic amino acids and the disulfide linkages. Figure 2B shows that the spectrum of native conalbumin in $0.1 \mathrm{M}$ sodium phosphate, $\mathrm{pH} 8.0$, consisted of negative extrema at $270 \mathrm{~nm}, 275 \mathrm{~nm}, 282 \mathrm{~nm}, 285 \mathrm{~nm}, 290$ $\mathrm{nm}$, and $295 \mathrm{~nm}$ and shoulders at 256 and $263 \mathrm{~nm}$, and of a positive extremum at $251 \mathrm{~nm}$. This spectrum was quite similar to those data reported. ${ }^{12,13)}$ The obvious difference in CD which showed negative values at below $260 \mathrm{~nm}$ and positive values at the region of $260 \sim$ $300 \mathrm{~nm}$ was seen between the native and the reacted conalbumin. Thus, these results indicate that during the 15-min incubation the conalbumin has a prominent alteration in the tertiary structure, but not in the secondary structure.

\section{Transmission electron microscopy}

The transmission electron micrographs of the reaction mixture at two different times are displayed in Fig. 3. At $17 \mathrm{~min}$ of incubation, when the turbidity of the sample was 0.02 at 

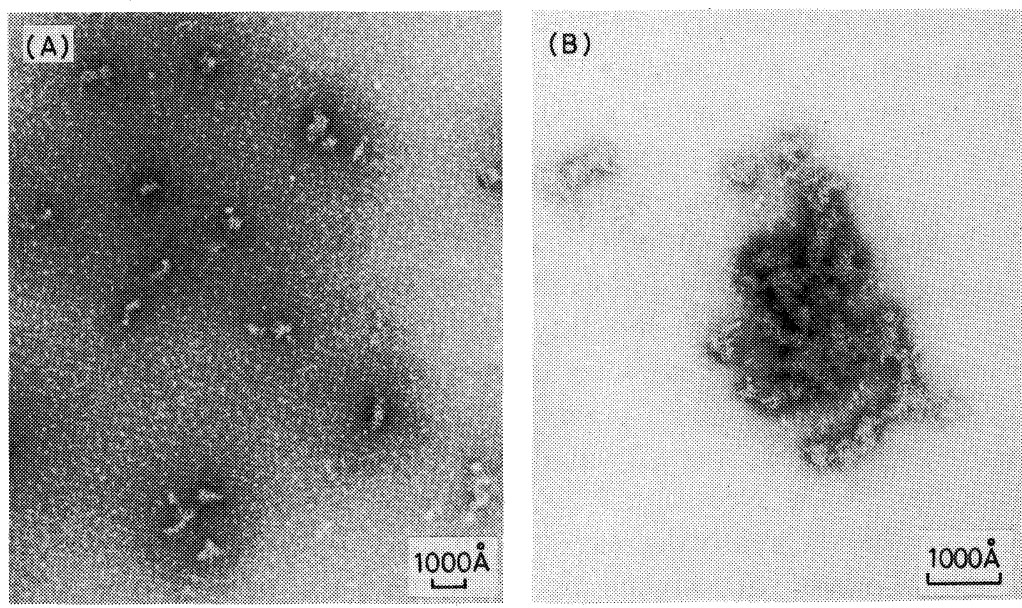

FIG. 3. Transmission Electron Micrographs of the 2-ME-Reacted Conalbumin.

After incubation with $70 \mathrm{~mm} 2-\mathrm{ME}$ at $35^{\circ} \mathrm{C}$ for $20 \mathrm{~min}$ (A) or $32 \mathrm{~min}(\mathrm{~B})$, the sample was diluted 80 times with distilled water and stained with $2 \%$ potassium phosphotungstate, $\mathrm{pH} 7.0$.

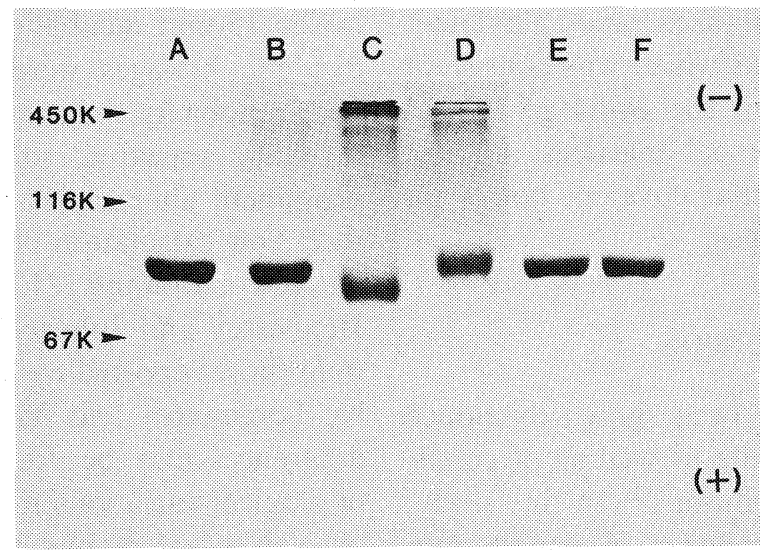

FIG. 4. SDS-Electrophoresis on Polyacrylamide Gel of Conalbumin.

Native conalbumin (lane A) and the conalbumin gel of $24 \mathrm{hr}$ incubation (lane B) were heated at $100^{\circ} \mathrm{C}$ for $2 \mathrm{~min}$ in the presence of $0.7 \mathrm{M} 2-\mathrm{ME}$ and $1 \% \mathrm{SDS}$. The same gel was dissolved in $0.1 \mathrm{M}$ sodium phosphate, $\mathrm{pH}$ 8.0 , containing $8 \mathrm{M}$ urea at $35^{\circ} \mathrm{C}$ for $10 \mathrm{~min}$ (lane C), and then treated with $2 \mathrm{~mm}$ iodoacetate for one hour (lane D). The 2-ME reacted conalbumin for $35 \mathrm{~min}$ (lane E) and $80 \mathrm{~min}$ (lane F) was dissolved in $0.1 \mathrm{M}$ sodium phosphate containing $8 \mathrm{M}$ urea and treated with iodoacetate in the same way. The proteins were electrophoresed on $10 \%$ polyacrylamide at $100 \mathrm{~V}$ for $6 \mathrm{hr}$ and stained with Coomassie blue.

$600 \mathrm{~nm}$, a structure $160 \AA$ in diameter and $350 \AA$ in length was observed with a relatively low frequency. The sample obtained at 20 -min incubation, with a turbidity of 0.05 , showed a rod-like structure of $260 \sim 300 \AA$ in diameter and $600 \sim 1,200 \AA$ in length (Fig. $3 \mathrm{~A})$. Thereafter, these structures increased with increasing turbidity, and various shapes connected with each other, apparently at random, were observed (data not shown).
At 32 min of incubation the sample with the turbidity of over 0.3 showed some branched aggregates (Fig. 3B).

\section{SDS-polyacrylamide gel electrophoresis}

We examined whether intermolecular covalent interactions including disulfide linkages among conalbumin molecules are involved in the thiol-dependent gelation. The turbid samples (35-min and 80-min incubations) or the 
conalbumin gels (24-hr incubation) were dissolved in $8 \mathrm{M}$ urea, then they were analyzed by polyacrylamide gel electrophoresis (Fig. 4). When the gel was dissolved in the presence of $2-\mathrm{ME}$ and $1 \%$ SDS, a single band was observed, which showed the same mobility as native conalbumin. The gel sample dissolved in $8 \mathrm{M}$ urea had a main band with mobility similar to the native conalbumin and several minor bands with higher molecular weights. However, when the gel sample was alkylated with iodoacetate before the electrophoresis, the contents of minor bands greatly decreased. Therefore, the minor bands with higher molecular weights appear to be caused by the formation of disulfide linkages during the gel solubilization or electrophoresis. When the turbid sample (35-min incubation or $80-\mathrm{min}$ incubation) was solubilized and alkylated, there was a single band which was consistent with the main band of the 24-hr gel. These results show that the gel matrices are not formed by intermolecular covalent bonds including disulfide linkages.

\section{DISCUSSION}

Thiol reagents have been shown to induce the gelation of egg white as well as of conalbumin under mild conditions. ${ }^{1)}$ In this study, we investigated the changes in conalbumin structure during the incubation with 2-ME to elucidate the gelation mechanism of this protein. There are fifteen disulfide linkages in the conalbumin molecule. ${ }^{2)}$ The disulfide linkages of conalbumin were cleaved with the addition of $70 \mathrm{~mm} 2-\mathrm{ME}$, and the sulfhydryl content almost linearly increased for $50 \mathrm{~min}$ with the reaction time (Fig. 1). The relative hydrophobicities estimated by both aliphatic and aromatic probes increased in parallel with progressive cleavage of disulfide linkages until $30 \mathrm{~min}$ of incubation. After a lag of about $20 \mathrm{~min}$, the turbidity of the conalbumin sample increased. From the transmission electron microscopic study, some structures which had dimensions of $160 \sim 300 \AA \times 350 \sim 1200 \AA$ were observed with relatively low frequency at 17 min of incubation (Fig. 3). These may be the polymeric forms of denatured conalbumin, since the native conalbumin has been described as an ellipsoid with dimensions of $98 \AA \times 60 \AA \times 40 \AA$ by $\mathrm{X}$-ray diffraction ${ }^{14)}$ and of $68 \AA \times 21 \AA$ by biophysical measurements. ${ }^{11)}$ These data strongly suggest that conalbumin is denatured by the 2-ME-dependent cleavage of disulfide linkages, which is consequently accompanied by the increase in hydrophobicity. The denatured conalbumin molecules may be polymerized with each other through intermolecular hydrophobic interactions.

To define the changes in conalbumin conformation during the incubation with 2-ME, we did a CD study (Fig. 2 and Table I). The CD data in the $190 \sim 245 \mathrm{~nm}$ regions indicated that in the 15-min incubation, when more than 2 disulfide linkages were cleaved, the relative hydrophobicity significantly increased, but no turbidity appeared, so this does not induce prominent alterations in the secondary structure. Our calculation of the $\alpha$-helix content of native conalbumin $(21.2 \%)$ by the method of Hennessey and Johnson ${ }^{6)}$ was slightly higher than those reported by Yeh et al. $(11 \%)^{11)}$ and by Tomimatsu and Vickery $(16 \%) .{ }^{12}$ These differences may be attributable to the methodological variations for calculation; the data of Yeh et al. ${ }^{11)}$ has been obtained by the method of Greenfield and Fasman, ${ }^{15)}$ and the data of Tomimatsu and Vickery ${ }^{12)}$ by the method of Chen and Yang. ${ }^{16)}$ The observed profiles of $\mathrm{CD}$ spectra of conalbumin were more closely related to the CD spectra obtained by programmed calculation using the method of Hennessey and Johnson than using the two other methods.

The CD spectrum in the $245 \sim 340 \mathrm{~nm}$ regions, especially at $250 \mathrm{~nm}, 267 \mathrm{~nm}, 275 \mathrm{~nm}$, $285 \mathrm{~nm}$, and $295 \mathrm{~nm}$, changed greatly during the 15-min incubation (Fig. 2B). Tan has reported that in this region the extrema at $291 \mathrm{~nm}$ and $295 \mathrm{~nm}$ are due to tryptophanyl residue of conalbumin, the extrema at $276 \mathrm{~nm}$ and $286 \mathrm{~nm}$, to the protonated tyrosine and the extremum at $251 \mathrm{~nm}$, to the disulfide link- 
age. ${ }^{13)}$ According to the data of Horwitz et al. ${ }^{17)}$ the change at $267 \mathrm{~nm}$ may be due to phenylalanine. The observed changes in the $245 \sim 340 \mathrm{~nm}$ regions agreed well with the increases in the sulfhydryl content and relative hydrophobicity. Therefore, the data of CD show that at least within $15 \mathrm{~min}$ of incubation the cleavage of disulfide linkages are accompanied by alterations in the tertiary structure but not in the secondary structure of conalbumin.

The conalbumin molecule consists of two domains, the $\mathrm{C}$ - and the $\mathrm{N}$-domains which are homologous in primary structure with each other. ${ }^{2)}$ Twelve among the fifteen disulfide linkages are at homologous locations in the two domains and the remaining three are in the C-domain. ${ }^{2)}$ The sulfhydryl content in the conalbumin gel was about 18 moles per mole of protein. For the iron-saturated conalbumin, Williams et al, have recently demonstrated the selective cleavage of only one disulfide linkage between residues 478 and 671 in the $\mathrm{C}$-domain by $5 \%$ dithiothreitol. ${ }^{18)}$ Our previous report has shown that the thiol-dependent gelation is completely inhibited by saturating conalbumin with iron. Therefore, it is very likely that most, if not all, of the nine disulfide linkages, which are cleaved by 2-ME in the absence of iron, are located near iron-binding sites and are important for the conalbumin gelation.

\section{REFERENCES}

1) M. Hirose, H. Oe and E. Doi, Agric. Biol. Chem., 50, 59 (1986).

2) J. Williams, T. C. Elleman, I. B. Kingston, A. G. Wilkins and K. A. Kuhn, Eur. J. Biochem., 122, 297 (1982).

3) G. L. Ellman, Arch. Biochem. Biophys., 82, 70 (1959).

4) L. A. Sklar, B. S. Hudson and R. D. Simoni, Biochemistry, 16, 5100 (1977).

5) E. Daniel and G. Weber, Biochemistry, 5, 1893 (1966).

6) J. P. Hennessey Jr. and W. C. Johnson Jr., Biochemistry, 20, 1085 (1981).

7) U. K. Laemmli, Nature, 227, 680 (1970).

8) O. H. Lowry, N. J. Rosebrough, A. L. Farr and R. J. Randall, J. Biol. Chem., 193, 265 (1951).

9) R. C. Woodworth and A. L. Schade, Arch. Biochem. Biophys., 82, 78 (1959).

10) S. Hayakawa and S. Nakai, J. Food Sci., 50, 486 (1985).

11) Y. Yeh, S. Iwai and R. E. Feeney, Biochemistry, 18, 882 (1979).

12) Y. Tomimatsu and L. E. Vickery, Biochim. Biophys. Acta, 285, 72 (1972).

13) A.-T. Tan, Can. J. Biochem., 49, 1071 (1971).

14) J. E. Abola, M. K. Wood, A. Chweh, D. Abraham and P. D. Pulsinelli, "The Biochemistry and Physiology of Iron," ed. by P. Saltman and J. Hegenauer, Elsevier, New York, 1982, p. 27.

15) N. Greenfield and G. D. Fasman, Biochemistry, 8, 4108 (1969).

16) Y.-H. Chen and J. T. Yang, Biochem. Biophys. Res. Commun., 44, 1285 (1971).

17) J. Horwitz, E. H. Strickland and C. Billups, J. Am. Chem. Soc., 91, 184 (1969).

18) J. Williams, K. Moreton and A. D. J. Goodearl, Biochem. J., 228, 661 (1985). 